\title{
Rites de passage à Wallis
}

Succession des générations et renouvellement de la vie

\section{Sophie Chave-Dartoen}

\section{(2) OpenEdition}

Journals

Édition électronique

URL : http://journals.openedition.org/jso/554

DOI : $10.4000 /$ jso. 554

ISSN : $1760-7256$

Éditeur

Société des océanistes

Édition imprimée

Date de publication : 1 décembre 2006

Pagination : 77-90

ISSN : 0300-953x

\section{Référence électronique}

Sophie Chave-Dartoen, «Rites de passage à Wallis », Journal de la Société des Océanistes [En ligne], 122-123 | Année 2006, mis en ligne le 01 décembre 2009, consulté le 30 avril 2019. URL : http:// journals.openedition.org/jso/554; DOI : 10.4000/jso.554 


\title{
Rites de passage à Wallis. Succession des générations et renouvellement de la vie ${ }^{1}$
}

\author{
par
}

\author{
Sophie CHAVE-DARTOEN*
}

\section{RÉSUMÉ}

La question des " rites de passage » est généralement considérée sous l'angle des spécificités de leur agencement ou de leur fonction sociale. Le problème est repris ici, à partir de l'étude des rites de mariage et de naissance à Wallis, pour montrer que ce qui importe, dans le système rituel que forment à Wallis les différents rites du cycle de vie, c'est moins le destin individuel, ou la reproduction de groupes sociaux antagonistes, que le renouvellement de la société elle-même à travers la prise en charge du premier enfant qui naît d'un couple.

Mots-CLÉs : Wallis (Uvea), rites de passage, primogéniture, ancienneté, mariage, naissance, communion.

Dans un effort de théorisation et de généralisation sans précédent, Arnold Van Gennep (1909) a proposé de définir les rites caractérisés par la succession de trois séquences (séparation, marge, agrégation) comme des « rites de passage ». Ces rites, souligne Arnold Van Gennep, marquent un changement

\section{ABSTRACT}

Rites de passage are generally studied for their specific organization or for their social function. In this paper I want to take this point further discussing a research carried out on Wallis island and looking at birth rituals and weddings. What is at stake during these life cycle rituals is less individual destiny or reproduction of antagonistic social groups than renewal of society itself through the caring of the first born child.

KeYwORDS : Wallis Is. (Uvea), rites de passage, primogeniture, wedding, birth, communion.

d'état ou de statut et, dans de nombreux cas, s'insèrent dans les rites du cycle de vie qui assurent le développement social de la personne selon une succession d'étapes propre à chaque société.

Ce mode de description et d'analyse, mettant l'accent sur le passage d'une "personne

1. L'essentiel des données ethnographiques sur lesquelles repose ce travail provient d'un terrain de dix-huit mois (1992-1994) à Wallis. Cette étude a été facilitée par les financements accordés par la Direction de la recherche et des études doctorales, le GDR 748 du CNRs et la Fondation de la Maison des sciences de l'homme. Le thème et l'argument de cet article découlent directement de ce terrain effectué, pour l'essentiel, dans la communauté d'Utufua, village situé au sud du district méridional de Mu'a. Qu'elle soit ici remerciée pour son accueil chaleureux et généreux. Toutefois, l'idée défendue ici selon laquelle ce qui fait l'objet des rites dits « de passage » n'est pas tant leur destinataire que les relations sociales qui, dans le monde austronésien, définissent et constituent la personne humaine, traverse toute l'œuvre de Daniel de Coppet et fut exposée en séminaire à l'EHESS au cours de l'année 1992. Ainsi, ce travail est dédié à Daniel de Coppet et à Aliki Liufau, référence en matière de « traditions » (aga'i fenua), à plusieurs reprises cité dans ce travail.

* Université de Bordeaux II, sophiedartoen@club-internet.fr 
focale $^{2}$ » et son intégration à la communauté, fut très largement adopté et, dans l'ensemble, peu discuté, si ce n'est dans deux contributions théoriques qui apportent un important contrepoint à cette perspective. Dans la première, Victor Turner (1969), en définissant comme non différenciées et non hiérarchiques les relations sociales en période de marge, montre que cette période peut se prolonger au point de devenir l'état particulier (appelé communitas) qui s'oppose à celui de la société structurée et étroitement régulée. Dans ce cas, les rites de passage concernent moins directement la «personne focale » que les groupes de référence auxquels elle est ainsi arrachée et intégrée. Dans la seconde, Pierre Bourdieu (2001) note que certains rites de passage, qu'il nomme "rites d'institution », ne tendent pas tant à intégrer qu'à exclure ceux qui ne sauraient en bénéficier, organisant ainsi des relations de domination entre groupes sociaux. Ces deux approches tendent ainsi à se détourner de l'agencement interne des rites organisant le destin de la "personne focale " pour considérer, selon des approches théoriques distinctes, les rites de passage dans la relation structurale qui les lie à la totalité sociale où ils prennent place.

L'étude des rites de passage wallisiens permet de poser de nouveau le problème de la relation entre la personne pour qui les rites sont organisés et l'ensemble des relations sociales dans lesquelles elle s'insère et évolue. En posant la relation entre personne et société, le présent travail s'intéresse, moins aux caractères morphologiques ou à la fonction des rites de passage, qu'à l'organisation spécifique des relations sociales que ces derniers mobilisent, assurant le renouvellement de la société à travers la succession des humains et manifestant par là l'agencement spécifique de la société wallisienne et des valeurs qui l'animent.

\section{Rites de passage à Wallis}

Différents rituels wallisiens qui entrent dans la typologie classique des "rites de passage » rythment le cycle de vie ${ }^{3}$. Ils conjuguent des rites catholiques (prières, messes ou bénédictions) et des pratiques que les Wallisiens consi- dèrent comme "venant du temps d'avant» (mai te temi mu'a). Ces rituels forment deux grands ensembles :

- Les premiers, nommés «jour de bain » (aho ma'anu), marquent la naissance et les rites de puberté masculins et féminins. Ils terminent un temps de réclusion par une grande toilette, une distribution de vivres et un repas. Cette réclusion de plusieurs semaines a lieu dans un milieu considéré comme chaud et humide, proche dans ses caractéristiques de l'utérus maternel. À cette occasion, les composantes de la personne les plus directement liées au souffle de la vie sont rituellement travaillées et renforcées en relation à la terre natale et au groupe social maternel localisé (Chave-Dartoen, 2000).

- Les seconds, nommés « investitures » (fakanofo), marquent les communions, les mariages et l'accès à une charge. Ils manifestent par une grande distribution de vivres précédée d'une cérémonie du kava, un changement dans les relations entre la personne, la société et Dieu. Dans ce cas, c'est avant tout de statut dont il est question.

Les rituels du premier type relèvent de responsabilités et d'un domaine essentiellement conçus comme féminins, tandis que les seconds relèvent de responsabilités et d'un domaine essentiellement conçus comme masculins.

Tous les rites de passage, à Wallis, sont très fortement marqués par la primogéniture : il est de règle que les cérémonies organisées pour le premier-né soient plus importantes, plus complexes et plus longues que pour les puînés, quel que soit le statut social de la famille concernée 4 . Corollaire de cette règle, tout est fait pour que le premier-né communie ou se marie avant ses frères et sœurs, de telle sorte que les cérémonies organisées pour lui soient, non seulement les plus importantes, mais aussi, les premières organisées par ses parents.

Cette valorisation de la primogéniture n'est pas propre à la société wallisienne. On la connaît dans toute l'aire malayo-polynésienne et Wallis, sous cet aspect, apparaît comme un cas d'espèce. De même, les rites de passage du type « jour du

2. L'usage de cette locution, qui me paraît plus appropriée que toute autre, m'a été proposé par Denis Monnerie. Je l'en remercie ainsi que pour les autres suggestions qui ont contribué à l'amélioration de l'ensemble de ce travail. Merci également à Cécile Leguy-Diarra pour sa relecture attentive.

3. Le cycle de vie suit, de nos jours, le modèle chrétien : s'étendant de la naissance à la mort (c'est-à-dire au départ de l'âme vers Dieu), il paraît écourté au regard des représentations pré-chrétiennes qui donnaient autrefois aux défunts une vie post mortem dans le monde de la Nuit d'où provenaient, en retour, les enfants à naître, vie et mort formant un continuum le long duquel circulait l'ensemble des êtres formant la société.

4. À cette complexité relative, déterminée par la primogéniture, s'ajoute celle déterminée par le statut social des groupes concernés : les cérémonies sont complexes pour tous les premiers-nés, mais plus encore pour ceux d'ascendance prestigieuse. Bien qu'il ne soit pas traité ici, cet aspect de la question mérite d'être souligné. 
bain » et « investiture » se retrouvent, sous des variantes locales, dans toute la Polynésie occidentale et, là encore, l'ethnographie wallisienne montre la grande unité culturelle de cette région centrale du Pacifique.

J'ai montré ailleurs (Chave-Dartoen, 2000) l'étroite relation de la primogéniture avec le renouvellement de la société wallisienne: l'aînesse y apparaît comme une modalité de l'ancienneté qui organise la succession des générations, l'aîné des enfants constituant, dès sa naissance, le relais entre la génération des parents et celle appelée à lui succéder. Ainsi, la naissance du premier enfant d'un couple inaugure une nouvelle génération en reléguant ses parents dans celle, supérieure, des "anciens » (mātu'a). Aussi, cette perspective renverse-t-elle celle communément admise concernant les rites de passage marquant le cycle de vie : à Wallis, ces rites de passage, et particulièrement ceux organisés pour le premier-né, ne concernent pas tant directement la personne focale que ses parents et l'ordonnancement général de la communauté, ainsi réaménagée. Au centre de ces rituels, bien sûr, le nouveau-né, le communiant, les mariés ou le défunt, mais l'étude attentive des cérémonies ${ }^{5}$ montre que, dans les trois premiers exemples tout au moins, si le traitement rituel s'attache à une personne, c'est aussi et surtout la relation entre ses père et mère, ainsi que leurs côtés cérémoniels respectifs, qui est retravaillée et affirmée.

Cependant, tous les rites faisant l'objet de notre étude ne sont pas d'égale valeur aux yeux des Wallisiens : les rites de naissance apparaissent, dans cet ensemble, comme fondamentaux, mais une étude attentive de l'ethnographie d'une part, et l'appel à la mémoire d'informateurs âgés d'autre part, ont montré qu'ils ne pouvaient être étudiés distinctement des rites de mariage, mariage et naissance formant, en quelque sorte, une unité tout autant dans la cohérence interne des actes rituels que dans les représentations qui les organisent et les animent. J'aborderai également les rites accompagnant la première communion, normalement intercalée entre la naissance et le mariage, comme autrefois les rites de puberté, aujourd'hui très allégés, surtout pour les jeunes filles dont les premières menstrues ne font plus l'objet d'une réclusion et de prestations spécifiques.

\section{Cérémonies de mariage}

Depuis la christianisation de l'île (1842), toute union est d'abord soumise à l'agrément du clergé catholique, mais en cent cinquante ans, les cérémonies se sont transformées de telle sorte qu'on ne peut parler du «mariage wallisien » comme d'une cérémonie d'organisation stable dans le temps ${ }^{6}$. À l'époque de mon enquête (1992-1994), les Wallisiens s'accordaient toutefois sur le prototype a minima ${ }^{7}$ d'un mariage tel qu'il convienne aux "règles de la coutume" ('aga fakafenua). Les longs préparatifs et l'ensemble des cérémonies, souvent très complexes, qui accompagnent le mariage à Wallis, ne seront pas détaillés ici, seuls les éléments saillants étant retenus pour cette description rapide.

Suite à la demande en mariage et à la visite des jeunes gens chez le prêtre, une période de ban de quatre semaines est annoncée lors la messe dominicale. Elle est mise à profit pour les derniers préparatifs menés à bien au sein de la maisonnée ('api) des parents de chaque futur marié : chacun des deux pères rassemble les provisions de victuailles qui complètent les prestations cérémonielles masculines de porcs et d'ignames, tandis que dans les deux foyers, les mères fabriquent les cosmétiques qui s'ajoutent aux prestations féminines faites de nattes et de tissu d'écorce (tapa). Dans le même temps, toutes les personnes en relation cérémonielle avec le père ou la mère d'un des mariés (parents, affins, amis) préparent une prestation et viennent constituer, autour des aînés de leur branche généalogique, le groupe cérémoniel auquel s'associent progressivement groupes collatéraux ou alliés apportant leurs propres contributions sous la direction de leurs propres aînés. Ces groupes et leurs prestations, qui se constituent ainsi en cascade, affluent ensuite sous forme de délégations (fagona) vers les deux maisonnées : d'abord les parents et les affins les plus proches, ensuite les relations plus éloignées. Tous forment, au jour des cérémonies, les vastes groupes cérémoniels (kutuga) qui

5. Cet article se concentre sur certains passages du cycle de vie. Les investitures à des charges officielles et, surtout, les funérailles - ensemble complexe de rituels qui clôturent le cycle de vie selon des modalités et des principes particuliers - feront l'objet de publications séparées.

6. Ces changements sont dus à différents facteurs. L'un d'eux est l'influence directe du clergé qui, pendant longtemps, instaura des mariages collectifs en tout début d'année civile. Un autre facteur est la généralisation progressive d'une forme de mariage somptuaire à mesure que les richesses importées devenaient accessibles à l'ensemble de la population. Un autre enfin, corollaire du précédent, est une simplification croissante des cérémonies qui, autrefois, s'étendaient sur plusieurs semaines, alors que les moyens numéraires mobilisés sont de plus en plus conséquents.

7. La description donnée plus loin repose sur des faits vécus et observés à plusieurs reprises, ainsi que sur les nombreuses discussions dont les cérémonies de mariage ont fait l'objet, à ma demande ou de façon spontanée. 
actualisent, pour l'occasion, l'étendue de la parentèle et des ramifications que créent, autour de chaque marié, relations de collatéralité, de mariage, de co-résidence et d'amitié. Ces groupes cérémoniels, formés à l'occasion du mariage comme des autres rites de passage, vont toujours par paire, le "côté de l'homme" (kutuga o te tagata), c'est-à-dire du père dans ce contexte, venant toujours « faire face » $\left(f e^{\prime}\right.$ 'haga'i) à celui de la femme (kutuga o te fafine), ici la mère - pour cette raison, je privilégierai désormais l'usage des termes " côté cérémoniel ».

Le jour du mariage, les côtés cérémoniels paternel et maternel se réorganisent ( $c f$. Fig. 1 et 2). Après s'être fait face, ils se regroupent pour un nouveau face à face : le "côté de l'homme" ne désigne alors plus le côté du père de chaque futur conjoint, mais celui du marié lui-même, tandis que celui « de la femme » désigne désormais celui du côté de la mariée. Ainsi, au jour des grandes cérémonies, nourriture, vêtements et autres prestations que chaque côté devra présenter au futur conjoint et au groupe que forment dorénavant ses deux côtés cérémoniels rassemblés sont prêts. Ainsi, le jour du mariage voit, après la rencontre successive des côtés cérémoniels maternels et paternels des parents des mariés, puis des mariés eux-mêmes, le face à face des vastes côtés que tous les présents forment, d'une part, autour du garçon, d'autre part, autour de la fille.

Les cérémonies de mariage se caractérisent, outre la bénédiction religieuse, par un allerretour répété des jeunes gens et de leur côté respectif entre les deux maisonnées organisatrices. La fiancée est d'abord conduite chez son futur époux pour être habillée et parée de façon somptueuse avant son apparition à l'église, ses parents pouvant faire de même concernant leur futur gendre. Après la bénédiction, chacun est de nouveau changé avant que la mariée et son escorte ne se rendent chez le marié. Là, danses, cérémonie du kava et distribution cérémonielle se succèdent, les jeunes gens étant à plusieurs reprises changés et parés de vêtements neufs et somptueux. Les mêmes séquences se répètent ensuite dans le même ordre, une fois les mariés rendus dans la maisonnée du marié. Le soir venu, ce dernier quitte alors formellement ses proches en larmes car il part, dit-on, pour suivre sa jeune épouse.

Cet adieu n'a, dans les faits, rien de définitif. Il souligne toutefois une caractéristique essentielle du mariage : à Wallis, la résidence est préférentiellement uxorilocale, surtout dans les premiers temps de la vie commune ${ }^{8}$. La nuit de noces et la défloration de la mariée ont normalement lieu chez celle-ci, dans un espace clos, spécialement aménagé à l'usage du jeune couple. À partir de ce moment, la jeune épouse reste normalement enfermée chez elle durant plusieurs semaines, cette réclusion étant présentée comme propice à la conception du premier enfant. Ce type de pratiques est devenu rare, mais en général, le jeune couple reste quelque temps chez la femme (de quelques semaines à plusieurs années) avant de changer de résidence. Pour cela, on attend généralement au moins la naissance du premier enfant. Les informateurs s'accordent sur le fait, qu'avant l'ouverture de la maternité de Mata'Utu, les femmes accouchaient auprès de leur mère. Si elles s'en sont éloignées, les primipares viennent encore y passer les dernières semaines de grossesse. La plupart y retournent, accompagnées de leur enfant, pour une nouvelle réclusion d'environ un mois que clôt, pour la mère et l'enfant, toilette et distribution de vivres du «jour du bain » (aho ma'anu). Une femme agit ensuite plus librement pour les enfants suivants.

\section{Similitudes et dissemblances entre mariage et naissance}

Il convient de souligner ici que, lors de la réclusion, le flux des prestations fournies est renversé et doublement dissymétrique par rapport aux échanges du mariage. À l'occasion du mariage, en effet, le flux des prestations cérémonielles passe du côté de l'homme à celui de la femme qui est accueillie dans la maison, puis du côté de la femme à celui de l'homme de façon répétée : le flux est ici croisé et alterné. Pour la naissance du premier enfant, en revanche, les prestations ne sont plus croisées, mais internes à chaque coté : dans sa maisonnée d'origine, les proches de la jeune femme n'accueillent plus son époux, mais prennent en charge ses seuls soins personnels (toilette, linge, literie...). Aux proches de l'époux, en revanche, reviennent les soins, non pas de l'homme, mais de l'enfant qui, de plus, ne réside pas chez eux : tous les jours, la grand-mère et les tantes paternelles viennent s'assurer de son bien-être et de sa santé. Au père, enfin, incombe de participer activement à la nourriture de la mère et de l'enfant (l'alimentation de la mère favorise la lactation), cette participation commençant avec la prestation formelle que constitue, tout de suite après l'accouchement, la pré-

8. Autrefois, le jeune couple pouvait alterner sa résidence à plusieurs reprises avant que le mariage ne soit consommé, mais la défloration devait toujours se passer chez la jeune femme. 


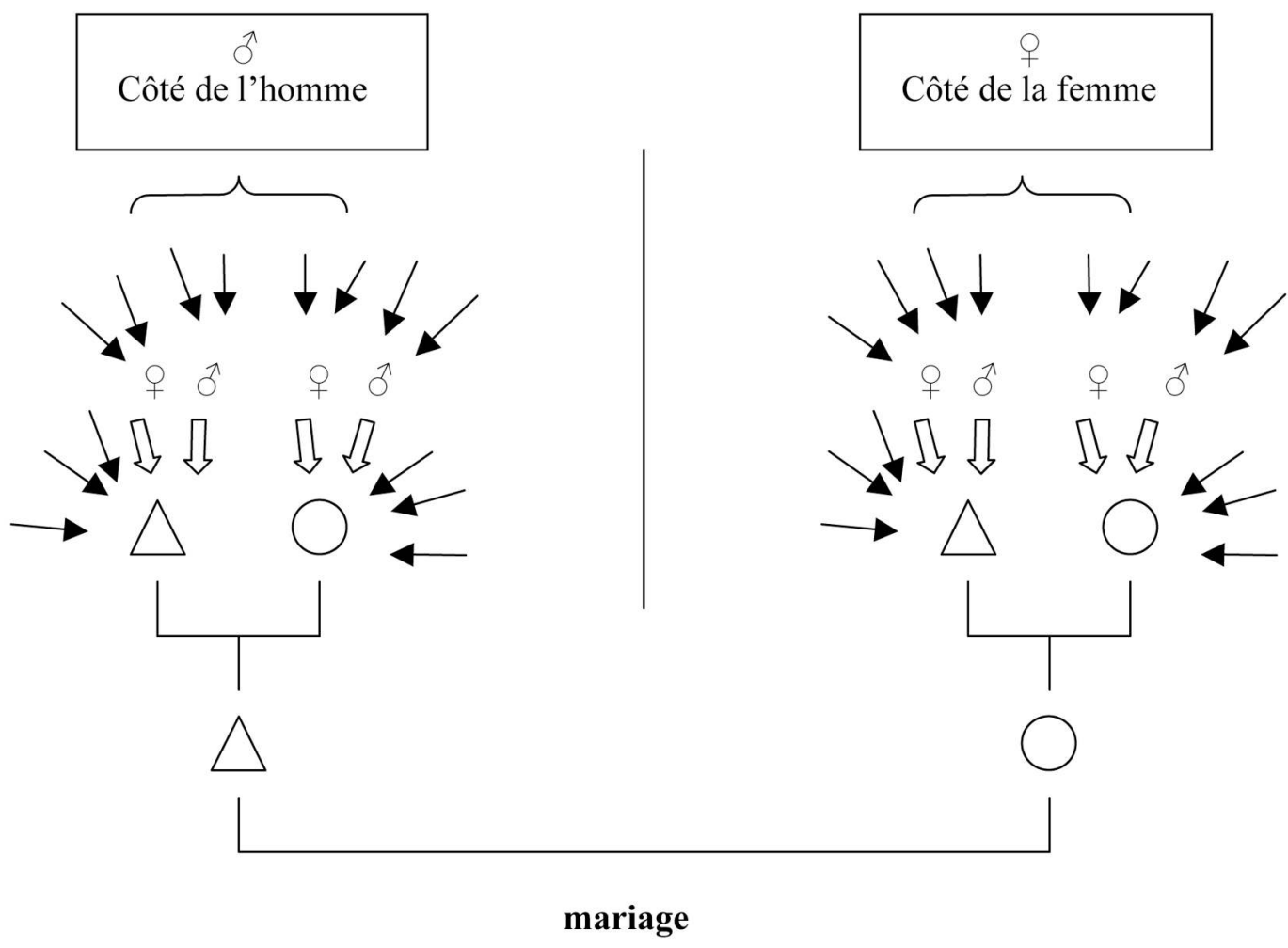

$\sqrt{3}$ prestations des côtés homme et femme à la génération des parents

prestations affluant des différentes branches collatérales à la génération des parents et des grands-parents
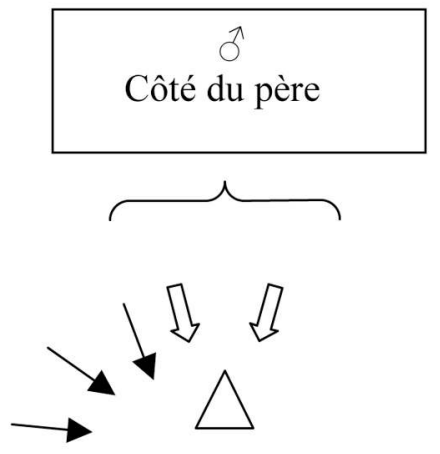

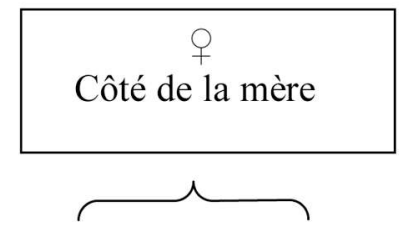

Côté de la mère

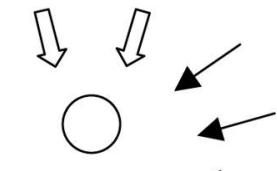

\section{naissance}

$\sqrt{3}$ prestations des côtés homme et femme à la génération des parents

/ prestations affluant des différentes branches collatérales à la génération des parents (prestations au niveau de la génération des grands-parents non représentées ici) 


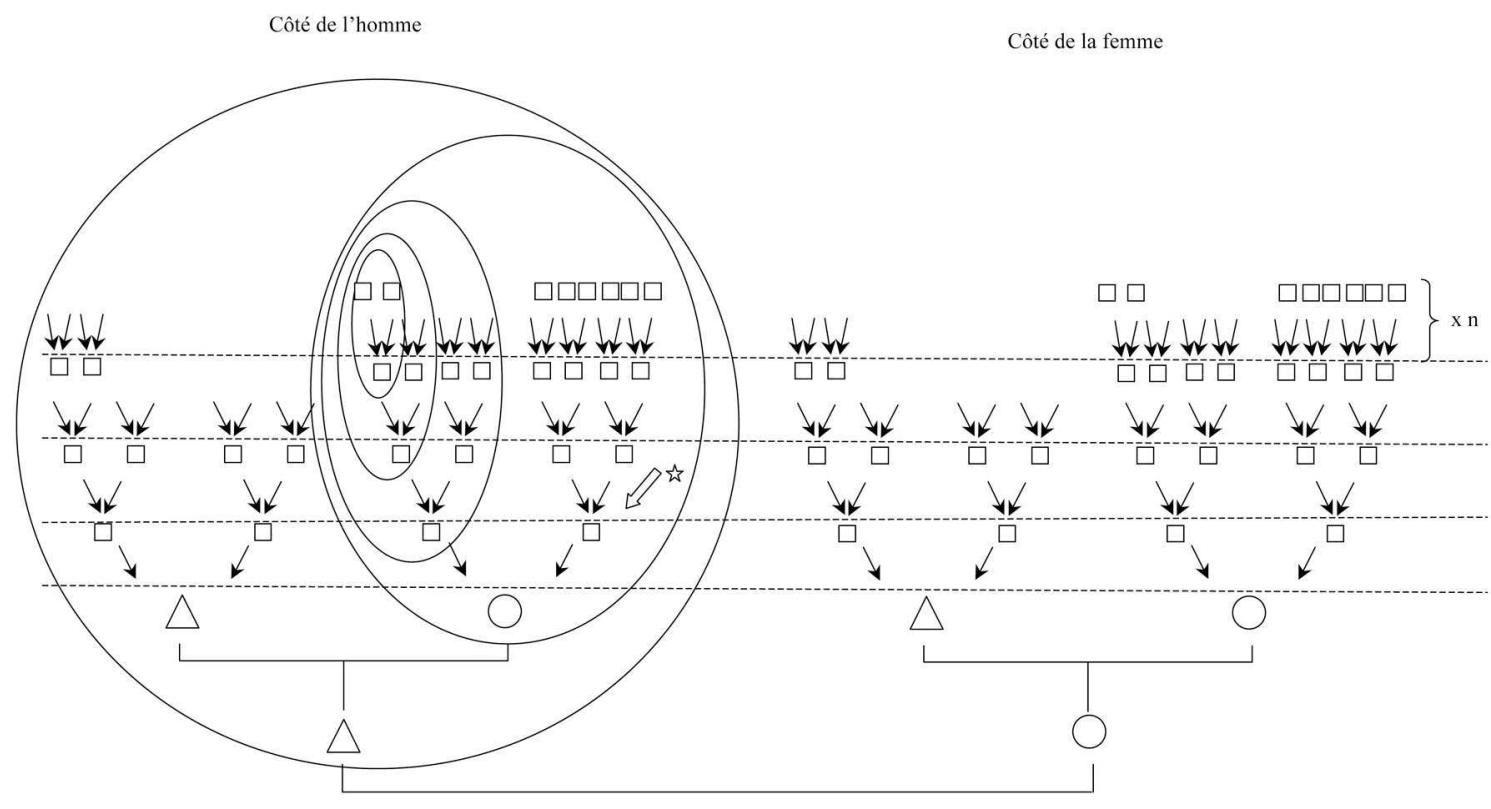

Exemple d'emboîtement des côtés cérémoniels et flux des prestations

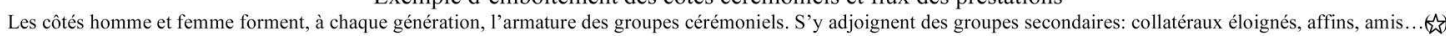

FIG. 2.

sentation du contenu d'un four ${ }^{9}$ dit « four de naissance » ('umu fanau) à la jeune mère.

À ces prestations, s'ajoutent, après un mois de réclusion, celles du « jour du bain » (aho ma'anu) qui lève les interdits, notamment sexuels, concernant la jeune mère. Pour l'occasion, la mère et l'enfant sortent devant la maison et président à une distribution de vivres (katoaga), le père de l'enfant et son côté apportant chez sa compagne des prestations masculines et féminines d'un volume exceptionnel. La nouvelle mère, qui a fortement grossi et dont le teint est devenu clair et lumineux, assiste alors à sa première messe, transformée dans son statut comme dans son apparence.

Les prestations présentées à l'occasion de la naissance et $\mathrm{du}$ « jour du bain » n'ont de réelle ampleur que dans le cas d'un premier-né. Pour les parents, une première naissance marque un passage important. Fatai Tui, une informatrice très âgée, expliqua qu'au "jour du bain », il s'agissait de " remercier [la mère] pour sa vie et son courage " (traduction littérale de fakamalo e kita ma'uli mo kātaki). Cette cérémonie, fait pendant au mariage qui l'a précédé, mais elle en diffère par une nouvelle dissymétrie. L'enfant prend ici, aux côtés de sa mère, la place du père dans une configuration où l'échange entre affins est amputé d'un de ses mouvements : si la parentèle de la mère ouvre sa maison sur l'extérieur pour accueillir sa contrepartie cérémonielle (le côté du père), les prestations ne sont pas formellement équilibrées comme lors du mariage. Dans ce cas, le "face à face » des côtés cérémoniels trouve une nouvelle expression, les groupes ne s'opposant plus de façon équilibrée (prestations tendant à l'équivalence), mais hiérarchisée : le côté maternel accueille et reçoit, le côté paternel arrive en donneur. Ici, la mère et ses proches constituent le côté maternel auquel l'enfant est rattaché et assimilé après une longue réclusion. $\mathrm{Au}$ «jour du bain», la mère et l'enfant sont récipiendaires des prestations portées par le seul " côté de l'homme », de telle sorte qu'à travers les prestations fournies à cette occasion, le côté du père vaut pour toute la parenté de l'enfant. Il le distingue des relations maternelles qui l'englobent et lui confère une forme d'existence plus complète (Chave-Dartoen, 2000).

La cérémonie " jour du bain » de naissance doit être rapprochée de deux occasions qui lui sont comparables sous différents aspects. En pre-

9. Les prestations masculines ('umu) sont principalement constituées du contenu d'un « four de pierre » (gutu'umu) creusé à même la terre où les vivres (un porc et des tubercules, éventuellement des préparations empaquetées dans des feuilles) sont cuits à l'étouffée. Dans le cas du «four de naissance », la prestation, une fois présentée à la parturiente par son conjoint, est ensuite divisée en trois parts : la sienne propre, celles de ses compagnes de chambre et celle du corps médical. Ces deux dernières parts sont à nouveau subdivisées. 
mier lieu, les Wallisiens eux-mêmes considèrent comme « identiques » (tatau) cette cérémonie de naissance et celle, de même nom (aho ma'anu), qui clôt la réclusion des garçons après leur circoncision. Dans les deux cas, explique-t-on, il s'agit d'honorer et de célébrer le courage dont le (ou la) récipiendaire a fait preuve sous la douleur et la saignée : l'écoulement de sang est, ici, mis en avant, quel que soit, par ailleurs, le comportement réel dans l'épreuve. Il s'agit là de marquer un passage sanglant, la première parturition étant pensée, pour les femmes ${ }^{10}$, comme équivalent (tatau) à la circoncision des hommes. Il est toutefois notable, qu'à la différence des femmes en couches, le lieu de la réclusion est indifférent pour les garçons à qui, autre dissymétrie, la prestation finale est offerte par leur propre groupe (" côtés » paternel et maternel rassemblés).

Par ses caractéristiques, la cérémonie "jour du bain » (aho ma'anu) des parturientes (réclusion chez les parents et prestation finale apportée par les affins) est proche, également, de la cérémonie (fua ta'ahine) qui suit normalement la défloration de la mariée. Suite aux cérémonies de mariage, la première relation sexuelle doit avoir lieu chez la jeune femme par "respect» (faka'apa'apa) pour elle. À l'aube qui suit la défloration, le jeune homme rapporte discrètement chez lui le drap nuptial, ses proches le raccompagnant chez sa compagne avec une importante prestation de biens masculins et féminins. Ici encore, une prestation fournie par l'homme et son côté suit une réclusion et un écoulement de sang pour la femme.

Cette description succincte du mariage et des pratiques accompagnant une première naissance visent à mettre en évidence plusieurs points qui doivent être développés dans les pages qui suivent afin de dégager les caractéristiques des relations que le mariage met en place à Wallis.

\section{Mariage et organisation villageoise}

Le mariage apparaît comme une occasion de mobiliser la parentèle étendue à travers la convergence et la redistribution de prestations dont les séquences sont identiques de part et d'autre et, au final, équilibrées. Des disparités en volume ou en qualité peuvent se manifester, à l'occasion d'un mariage, selon l'étendue des parentèles mobilisées et les moyens dont leurs membres disposent. Cependant, chaque côté met un point d'honneur à fournir des prestations remarquables de façon à ne pas perdre la face. Dans cet agencement, il faut souligner toutefois quelques rares dissymétries très significatives. Elles mettent en œuvre deux principes.

Certaines prestations n'ont pas de contrepartie exactement symétrique - demande en mariage, prestation supplémentaire lors du repas chez le marié, constitution de la couche nuptiale... Dans ce cas, la distinction de sexes est pertinente en ce que, pour l'essentiel, le côté du garçon fournit des biens masculins à celui de la fille, tandis que celui de la fille présente des biens féminins à celui du garçon, les deux côtés paraissant ici complémentaires et non compétiteurs.

L'autre dissymétrie consiste en ce que, pour la demande en mariage, le mariage lui-même, la défloration puis, la naissance, le côté de la femme accueille toujours, in fine, celui de l'homme, ce qui est cohérent avec l'uxorilocalité préférentielle.

Il apparaît ainsi que le mariage (oho'ana) associe, au-delà du " couple » (tau matu'a) formé d'éléments différents et complémentaires, deux ensembles de relations marqués, l'un comme féminin ("côté de la femme »), l'autre comme masculin ("côté de l'homme») et non interchangeables. Il est révélateur à cet égard que les dissymétries concernant le mariage se retrouvent dans les rites de naissance, les cérémonies qui encadrent la première grossesse (mariage, défloration et naissance) présentant une grande cohérence globale.

Le couple forme donc une unité stable dont les membres, nettement différenciés, sont destinés à prendre en charge une "maisonnée " ('api). Celle-ci constitue une unité sociale qui associe le travail et les responsabilités des deux époux afin de fournir une prestation appropriée - biens féminins et masculins associés - en toute circonstance cérémonielle: obligations villageoises, mais aussi et surtout, nous l'avons vu, participation aux cérémonies concernant la parentèle de chacun des membres du couple. Le mariage initie donc l'intégration des jeunes époux à la communauté sous une nouvelle forme. Dans les six mois qui suivent le jour des noces, ils passent du statut de célibataires appartenant au groupe des « enfants» du village (tamaliki eva « enfants sans attaches »), groupe social où les filles four-

10. Lors de leurs premières règles, les jeunes filles subissaient autrefois également une réclusion suivie de prestations, mais d'une importance bien moindre que celles des cérémonies aho ma'anu telles qu'on me les a décrites. Ces pratiques ont été abolies par les religieuses qui, dès la fin du $\mathrm{XIX}^{\mathrm{e}}$ siècle et pour plusieurs dizaines d'années, ont ouvert des internats (nofokolo) où toutes les filles célibataires devaient venir dormir. Actuellement, les jeunes filles restent chez elles un ou deux jours pour leurs premières règles, buvant des boissons chaudes et évitant de se baigner comme leurs aînées en période de menstrues. 
nissent collectivement des prestations féminines, les garçons des prestations masculines, à celui d'«anciens» (mätu'a) qui, en couple, sont capables de fournir une prestation complète. Cette période de latence voit donc s'initier la grossesse de la jeune femme et l'agrégation progressive du jeune couple à l'assemblée des « anciens » du conseil de village où il leur faudra progressivement gagner la considération et le respect de leurs pairs. À terme, le jeune couple prend ainsi en charge la gestion d'une maisonnée. Ce faisant, il assure la pérennité de l'organisation villageoise qu'il maintient en prenant le relais de ses propres anciens, non seulement dans la gestion de cette maisonnée, mais aussi dans l'occupation des terres familiales et les responsabilités cérémonielles.

À cet égard, toutefois, il est important de souligner que la descendance n'est pas une condition sine qua non pour l'accès au conseil et au statut implicite de matu'a. De même, chose fondamentale, une résidence n'est pas nécessairement partagée entre parents et enfants ou transmise par voie de filiation. Ainsi, l'engendrement fonde seulement en partie le statut d' " ancien » dans la communauté villageoise tout comme, dans une perspective inversée, la descendance directe donne un accès facilité, mais non exclusif, aux terres ancestrales. Dans les deux cas, la procréation favorise, sans la conditionner, la perpétuation de l'organisation sociale. Insistons sur le fait que, si, ici, la succession est formulée en termes de parenté ${ }^{11}$, il s'agit d'un usage particulier de ces termes, la référence à une notion de parenté très englobante prenant un caractère métaphorique à l'échelle du village et classificatoire à l'échelle de la maisonnée ${ }^{12}$.

Le mariage apparaît donc comme un dispositif de relais dans la prise en charge d'une unité sociale sur une terre où le principe de permanence et la force des relations cérémonielles ainsi constituées et reconduites priment sur celui de continuité généalogique ${ }^{13}$. Dans ce dispositif assurant le renouvellement de la vie et le prolongement des activités cérémonielles de la maisonnée, l'enfantement est secondaire.

Les rites accompagnant la naissance d'un premier-né mettent toutefois en place un autre type de dispositif, qu'il convient de préciser. Lors de la naissance, les prestations des paternels (réclusion et «jour du bain») mettent en évidence l'importance de la relation de germanité de sexe opposé pour la vie et le statut des enfants d'un homme. Si le père prend en charge la nourriture de la mère et de l'enfant, sa propre mère et, surtout, ses sœurs prennent soin de l'enfant d'une façon très attentive. À défaut, refus ou négligence seraient interprétés comme une suspicion sur l'ascendance paternelle de l'enfant, entachant sa naissance du doute d'une possible bâtardise. Plus grave encore, dans un contexte de tension dénué de ce type de suspicion, toute mauvaise volonté apparaîtrait comme une manifestation de ressentiment ou de colère et, venant des tantes paternelles, comme une mise en danger réelle et immédiate de la vie de l'enfant. Les cas de maladies graves et de décès d'enfants imputés au ressentiment (lotokovi) d'une tante paternelle sont nombreux à Wallis, cette capacité à tuer étant la contrepartie de la garantie de vie et de santé que la sœur du père assure pour ses neveux agnatiques en relation à ses ancêtres et à Dieu.

On le voit ici, la relation de germanité de sexe opposé constitue donc, à travers la paire frèresœur, une forme de relais, dans la transmission de la vie, qui ne s'actualise pas dans la gestion et le renouvellement d'une maisonnée, mais dans la proximité et la continuité qu'elle assure en relation aux ancêtres paternels et à Dieu. Ce type de responsabilité se manifeste clairement lorsque, une fois les parents morts, les aînés de chaque

11. Les termes de parenté désignent les relations entre villageois, selon des modalités différentes, en fonction du contexte. D'abord, dans le discours courant, les villageois s'organisent en deux ensembles complémentaires, les « anciens » mätu'a et les « descendants » fanau, cette opposition organisant, en amont, tout le système des classes d'âge. Puis, dans les discours formels, les intervenants classent ceux à qui ils s'adressent parmi les pères et mères, les germains de même sexe ou de sexe opposé ou encore les descendant masculins ou féminins selon leurs âge et sexe. Enfin, la femme du chef est appelée " mère du village », le chef, de façon significative, « ancien (et non père) du pays » (matu'a fenua), les villageois formant une " grande parenté »fo' $i$ kaiga.

12. L'usage du mot « parenté » est mal approprié pour définir ce type de relations car les Wallisiens font rentrer les relations de parenté dans un cadre beaucoup plus vaste que celui défini par les domaines conjugués de la consanguinité et de l'affinité. Le terme kaiga, traduit en français par "parenté », désigne le fait de partager des relations cérémonielles, la nature et le degré de la relation étant spécifiés subsidiairement : kaiga toto « relation de sang », kaiga totonu « relation vraie », kaiga fai « relation faite $\gg . .$.

13. Le «partage » des droits sur la terre au profit de personnes n'ayant pas de droits reconnus en vertu de relations généalogiques est fréquent et fait l'objet de différentes modalités, transfert d'enfant compris. Dans une logique similaire, les droits de personnes établies de longue date sur une terre d'habitation sont considérés comme plus légitimes que ceux des absents, quand bien même les relations généalogiques à l'ancêtre de référence sont beaucoup moins directes dans un cas que dans l'autre. Selon Aliki Liufau, le fait de «vivre d'une terre» (ma'uli i te kele) était autrefois constitutif des droits que l'on pouvait faire valoir à son égard. 
sexe s'associent - en tant que paire de germains de sexe opposé - et leur succèdent dans l'organisation des prestations au niveau de la branche généalogique dont ils prennent la tête et dont ils organisent l'ensemble des activités cérémonielles. Ainsi, tandis que le couple assure de son vivant l'organisation de la maisonnée, la paire de germains aînés de sexe opposé forme l'armature autour de laquelle s'arriment les relations généalogiques et cérémonielles, assurant l'organisation du côté cérémoniel kutuga suite au décès ou à l'incapacité du couple des parents.

Tels qu'ils apparaissent à l'observateur, les rites de mariage ne laissent toutefois pas bien voir comment s'articulent ces deux types de relations, d'une part entre elles et, d'autre part, respectivement avec le mariage. Il faut, pour mieux les comprendre, développer plusieurs faits décrits ci-dessus et relatifs à la naissance du premier enfant. Je vais y revenir, après avoir insisté sur l'importance de la prestation de naissance 'uти fanau et sur les rites de reconnaissance, actuels et anciens, auxquels elle renvoie.

\section{Rites de reconnaissance et constitution d'une descendance valorisée}

Pour chaque naissance, la prestation appelée "four de naissance " ('umu fanau) est impérative, que ce soit sur un mode formel, pour un premier-né, ou sur un mode plus informel, pour les enfants suivants. Un homme qui s'en dispenserait signifierait de façon publique le rejet d'un enfant qu'il considérerait comme n'étant pas le sien. Il est courant d'entendre louer, à Wallis, la générosité d'hommes qui, dans un mouvement inverse, acceptent de cette manière comme étant les leurs des enfants dont ils se doutent qu'ils sont adultérins. Aussi les Wallisiens admettentils couramment que la paternité se construit à travers l'alimentation et le travail que fournit un homme pour nourrir ses enfants. Avant d'être biologique, le père est avant tout nourricier, cette valorisation des relations socialement construites se trouvant, par ailleurs, au cœur des transferts d'enfants.

Il apparaît ainsi qu'à l'encontre du mariage contracté selon le droit civil français, le « mariage wallisien ${ }^{14}{ }^{14}$ 'instaure pas entre les deux époux un lien qui sera automatiquement transmis aux enfants. À Wallis, la relation père/enfant doit être, chaque fois, construite et affirmée entre le père et le nouveau-né, une relation de mariage préexistante n'étant pas suffisante à cet égard. Ainsi, pour chaque enfant, tandis que la relation à la mère (et au côté maternel de la parenté) est donnée, celle au père et aux paternels est construite : d'abord, lors de la présentation du " four de naissance »; puis, durant la réclusion de la mère et de l'enfant, par la prise en charge des soins et de l'alimentation de ce dernier par le père et ses proches, les sœurs du père en premier lieu.

La présentation d'un four de naissance revient donc à une "reconnaissance» (fakamo'oni), celle-ci étant nécessaire, que l'enfant soit légitime ou non. Dans le premier cas, la prestation va très généralement d'elle-même, le père apportant toujours de la nourriture lors des visites à la maternité. Dans le second cas, la prestation est très explicitement désignée par le terme fakamo'oni. Elle peut avoir lieu dès la naissance, mais peut aussi venir après un délai au cours duquel l'homme s'assure, en observant l'enfant et son développement (fili ki tu'a), qu'il en est bien le père. Cette prestation revient, pour le père et son côté, à « faire amende honorable » (fai hu) dans le cas où la naissance extra-conjugale ne pourrait être régularisée par un mariage hâtif. Elle est généralement consentie sous la pression de la future mère, de ses proches et du clergé qui mettent tout en œuvre pour obtenir une « réparation » (tu'aia) afin de « rembourser la virginité de la fille », de lui « rendre son honneur $»^{15}$. En cas de refus devant l'évidence, l'affaire est portée devant les chefs coutumiers à qui il revient d'imposer une amende dont ils fixent le montant. À noter qu'une fois l'enfant reconnu (la présentation de la prestation forme à la fois l'apex d'une crise et sa résolution), l'entente revient souvent entre les familles, le côté du père continuant de se manifester plus ou moins discrètement lors des cérémonies concernant l'enfant et ses proches. Avortements - autrefois par empoisonnement volontaire, de nos jours par IVG à Nouméa ${ }^{16}$ - et infanticides ne sont pas rares, dit-on, mais le relevé des généalogies villageoises montre que les enfants naturels sont nombreux. De statut inférieur aux enfants légitimes, ils sont généralement

14. La loi statutaire $n^{\circ} 61814$ du 29 juillet 1961, qui entérine le statut de том pour Wallis-et-Futuna, attribue la citoyenneté française aux insulaires des deux archipels, mais leur reconnaît un régime juridique de droit particulier : sur ces territoires, la chefferie est garante des droits et devoirs de chacun, elle seule fixe les règles de la « coutume » ('aga fakafenua).

15. Entretien avec le père Ikauno.

16. Les avortements sont clandestins ou secrètement organisés sur Nouméa du fait de l'interdit qu'y oppose l'Église, mais surtout afin de protéger, avec la réputation de la femme (il s'agit bien souvent de jeunes filles encore non mariées) et de son groupe, les chances d'une union honorable. Une étude statistique de ces pratiques montrerait certainement une différence de statut social entre les femmes mettant au monde des enfants naturels et celles ayant recours à des IVG discrètes. 
confiés à des proches par la mère si elle a, par la suite, l'occasion de se marier.

Une jeune femme est dite taupo'ou (nom désignant également les religieuses catholiques) dans la mesure où, vierge, elle est "sacrée » (tapu), c'est-à-dire « interdite, réservée ». C'est dans cet état que chaque famille veille à mener ses filles jusqu'au soir du mariage où, sous la surveillance des vieilles femmes, elles seront déflorées par leur époux. Tout comme le mot taupo'ou, répandu dans toute la région, cette pratique est bien antérieure à la christianisation de l'île, la virginité des jeunes filles étant considérée comme un « bien de valeur » (koloa) pour la famille.

La femme est considérée, à Wallis, comme garante de la fertilité du couple et sa virginité, dit-on, appelle la bénédiction de Dieu. La virginité de la mariée est aussi une garantie de loyauté, les femmes étant soupçonnées de ne pouvoir résister aux sollicitations d'anciens amants. Mais, tout comme les prestations de défloration (fua ta'ahine) préfigurent celles de la fin des couches, c'est certainement en relation à la première naissance que la virginité prend toute sa valeur. Le premier-né, issu de la "première ouverture " ('uluaki avahi) de sa mère, initie sa vie de femme et, ouvrant une génération, vaut pour tous les enfants à venir. Cet enfant, plus que tout autre, « revient à ses paternels » (e ma'u ia te kutuga a te tamai) qui le prennent en charge dès la naissance et lui donnent le nom d'ancêtres valorisés. Plus que tout autre enfant, cet enfant est complet, en relation directe et explicite avec les maternels, mais aussi et surtout avec les paternels qui, à travers les cérémonies auxquelles ils prennent part, lui donnent statut - accès prioritaire aux titres et au patrimoine de ses ancêtres et autorité sur les puînés à venir. Les prestations de défloration (fua ta'ahine) et de naissance ('umu fanau et aho ma'anu) manifestent ainsi des faits de deux ordres: premièrement le statut supérieur de la jeune femme arrivée vierge au mariage et de son groupe; deuxièmement, la force du lien qui, au-delà du couple qu'il forme avec une partenaire valorisée, s'établit entre un homme et les enfants issus de ce type d'union.

Dans cette configuration, cérémonies et relations de mariage sont à la fois importantes - elles forment le cadre de toutes les autres prestations et très subordonnées - elles ne suffisent pas à établir une relation satisfaisante entre un homme et les enfants de sa compagne. Pour comprendre cet agencement rituel particulier, qui vient doubler le sacrement du mariage chrétien par la reconnaissance des enfants qui en résultent, il faut considérer les pratiques matrimoniales antérieures à la christianisation. Les données à ce sujet sont rares, mais concordantes avec ce que l'on sait des sociétés proches à la même époque.

Un des rares témoignages publiés est celui du médecin de Marine Viala qui séjourna à Wallis au tout début du $\mathrm{Xx}^{\mathrm{e}}$ siècle.

« La polygamie était autrefois le seul régime matrimonial tout au moins pour les chefs, dont elle constituait une sorte de privilège ; les autres indigènes s'unissaient et se quittaient au hasard des circonstances : c'étaient plutôt des rencontres que des unions durables. » (1909: 254)

Les informations recueillies oralement auprès d'Aliki Liufau ont précisé ces faits. Il a retenu du témoignage de ses anciens qu'il n'y avait pas à proprement parler de cérémonie de mariage autrefois à Wallis. Ce qui comptait, à l'époque, c'était la prestation taulolo que le père présentait à la mère du nouveau-né : le contenu d'un four et des cosmétiques. Un homme pouvait choisir de reconnaître, chez ses compagnes simultanées ou successives, les seuls descendants qu'il agréait. De cette reconnaissance dépendait le statut de l'enfant, celui d'une femme aristocrate n'étant lui-même aristocrate que si un père de même statut l'avait reconnu. Cela n'excluait probablement pas les unions stables et la reconnaissance par un homme de la série des enfants nés de ses compagnes régulières, mais l'opération n'était ni automatique, ni systématique ${ }^{17}$.

Dans cette perspective, la virginité prend plus d'importance encore que dans le cadre du mariage chrétien. Aliki Liufau a insisté sur le fait qu'autrefois les unions respectaient une endogamie d'ordre social, signifiant par là que des partenaires ou des compagnons roturiers ne convenaient pas aux jeunes filles de haut statut ${ }^{18}$. L'inverse, en revanche, n'était pas tout à fait vrai,

17. Cela n'excluait probablement pas, non plus, une certaine forme d'union rituellement célébrée, tout au moins pour ce qui regardait les jeunes filles de haut statut. Le terme polo (promesse de mariage entre enfants de grandes familles) semble le confirmer, tout comme l'ancienneté attestée du terme oho'ana qui, de nos jours, désigne le mariage et les cérémonies de mariage. Un autre fait est significatif, l'importance de la virginité, surtout pour les jeunes femmes de haut statut, qui laisse à penser que la défloration ne devait pas se passer sans une cérémonie entérinant leur union avec des hommes de haut rang.

18. Ce clivage s'est estompé sous la pression insistante des missionnaires qui, combattant ce qu'ils considéraient comme les privilèges immoraux de l'aristocratie (polygynie, droit de vie et de mort sur les dépendants, perception de redevances journalières...), imposèrent le mariage catholique et favorisèrent, à chaque fois qu'ils le purent, les mariages mixtes. Les jeunes gens font désormais à leur guise, soutenus par le clergé, mais dans les discours, on dénote une nette valorisation de l'hypergamie. 
un «chef » accueillant dans sa résidence, outre sa ou ses compagnes attitrées, leurs suivantes et les femmes du peuple dont il lui plaisait de s'entourer (information d'Aliki Liufau). Nul doute que, dans ce contexte, seuls étaient reconnus les enfants nés des compagnes de haut statut qui, leur étant parvenues vierges, mettaient au monde les enfants que les aristocrates reconnaissaient pour descendants (et le premier-né au-dessus de tous). Il est probable que, dans ce cas comme de nos jours encore, le premier-né valait pour tous ses puînés, la virginité initiale d'une mère de haut statut garantissant au couple ainsi formé une descendance commune assurée par une relation privilégiée. Les traditions montrent que ces unions, valorisées pour la descendance ainsi constituée, n'étaient cependant pas nécessairement vouées à perdurer et qu'une fois des enfants nés, le couple pouvait se séparer pour habiter des résidences différentes ${ }^{19}$.

On le voit, ce qui était alors en jeu, pour les hommes comme pour les femmes, et bien au-delà d'eux pour leurs côtés respectifs, était de travailler au statut de chacun de leurs descendants afin de distinguer (faka'iloga), parmi les enfants, ceux qui seraient de haut statut 'aliki (reconnus par un père aristocrate) des autres (tu'a), non reconnus ou reconnus par un père roturier. Dans le premier cas, le statut se définissait par l'ajout d'une ascendance paternelle prestigieuse, et d'un côté cérémoniel extrêmement valorisé, à l'ascendance maternelle et au groupe dans lequel l'enfant naissait.

La cérémonie taulolo conférait à l'enfant une forme d'existence plus complète du fait d'une relation revendiquée par les paternels (le père, ses sœurs et leurs parents) qui assuraient ainsi son statut et sa position sociale. Cette cérémonie, donnant un père à l'enfant, unissait deux ensembles de parents, deux côtés, autour de lui plus qu'elle ne scellait une union durable entre ses parents. L'enfant était ainsi vu comme le point de jonction de réseaux de relations qui s'unissaient en lui, composant sa personne, formant sa « parentèle » kaiga et lui conférant un statut au cœur de ses deux « côtés cérémoniels 》 (kutuga) et de la société. Pour les enfants suivants, la cérémonie avait probablement moins de résonance dans la mesure où les parents continuaient à cohabiter. L'homme continuait à nourrir sa compagne durant la grossesse, ce qui revenait à une reconnaissance de fait, la cérémonie ultérieure étant purement formelle.

De nos jours, le mariage pérennise l'union des parents avant même la naissance du premier enfant et la cérémonie taulolo n'est plus pratiquée, mais un homme continue à fournir les prestations qui établissent avec ses enfants la relation de paternité telle qu'elle est socialement validée, que ces enfants naissent dans ou hors du mariage. Au-delà du lien entre un homme et une femme que ces cérémonies soulignent, c'est le lien entre deux ensembles de relations formés autour d'un enfant (ses deux kutuga) qui est constitué puis affirmé au fil des cérémonies successives. $\mathrm{Si}$, rappelons-le, le lien à la mère et à ses parents est admis comme allant de soi, le père et ses parents doivent, dans tous les cas, manifester et actualiser leur relation à l'enfant et à sa mère par des prestations répétées en nourriture et en biens féminins. Ce faisant, ils affirment la relation entre l'enfant, surtout un premier-né, et l'ensemble de ses paternels, reconnaissant en ce dernier, de par sa complétude achevée, un descendant d'une qualité particulière. Il est donc ici question de statut, celui de l'enfant, mais aussi et surtout celui de ses deux côtés cérémoniels. Les enfants nés d'unions prestigieuses accumulaient en leur personne (et accumulent encore), mais au profit de tout leur groupe, un réseau de relations à la fois denses et étendues tandis que, pour les enfants de statut inférieur, ces relations, à la fois peu étendues et moins denses, les associ(ai)ent à la masse moins différenciée de la population.

Rites de mariage et de naissance opèrent ainsi, au cas par cas, un travail sur les relations sociales mobilisées, évaluées et réajustées. Il nous reste toutefois à comprendre à quel niveau ce réajustement est significatif du point de vue de la société wallisienne. Est-ce à celui des enfants comme le laisse entrevoir l'importance du premier-né en qui se concentrent statut et autorité, ou à celui des parents, comme on peut le comprendre en considérant l'élévation sociale que la naissance d'un enfant de femme vierge reconnu par un homme de haut statut assure en retour à ses deux parentèles? Pour y répondre, il faut revenir à la valeur que les Wallisiens reconnaissent au premier-né et à ceux des rites de passage qui le concernent directement, à commencer par la première communion dont il n'a pas encore été question.

19. C'est ce que montre, entre autres exemples, le récit de Talapili et Talomohe où les parents de Toamisa, Lupeolo et Fuluhega résident séparément ( $c f$. Henquel, $m s$ et les entretiens avec Aliki Liufau). L'ensemble de ces faits est proche de ce que l'on sait concernant Tonga et Samoa à la même époque. À noter que si l'union à une fille vierge assurait aux aristocrates une descendance privilégiée, polygamie et reconnaissances tardives n'excluaient pas des rivalités entre les descendants de différents lits et d'éventuels réaménagements dans les généalogies prestigieuses. Le fait est attesté partout dans la région. 


\section{Primogéniture et rites de passage}

Les rites concernant le premier-né présentent aujourd'hui, à Wallis, des caractéristiques bien singulières. Mises à part les cérémonies de naissance, c'est lors de la première communion que la spécificité du premier-né est la plus évidente. La première communion commence par une courte retraite religieuse des enfants (fakamamahi), tandis que leurs familles, tout comme pour un mariage, commencent à rassembler les vivres et les biens de valeur nécessaires à l'accueil des participants et aux prestations distribuées pour l'occasion. Pour chaque enfant, les côtés cérémoniels des père et mère se forment de nouveau et affluent vers la maisonnée. Comme pour un mariage, l'étendue des côtés et l'importance de leur participation varient s'il s'agit d'un premier-né ou d'un puîné, mais la distinction essentielle réside dans la qualité de la cérémonie du kava qui suit la messe de communion. La célébration religieuse, en effet, est suivie d'une distribution de vivres précédée par une cérémonie du kava ou d'un toast au champagne. Ces dernières cérémonies ne sont toutefois pas de même qualité pour un aîné ou un puîné : ce dernier bénéficie d'une cérémonie informelle en présence du chef de village ou de son représentant, tandis que pour le premier, la cérémonie est réalisée, par autorisation expresse du « roi » (hau), en présence d'un «ministre » ('aliki fa'u) qu'il a délégué pour l'occasion. Cette distinction est fondamentale en ce qu'elle instaure une proximité statutaire - par délégation $\mathrm{du}$ « roi », c'est la coupe royale qui est présentée à l'enfant entre le premier enfant d'un couple et le « roi » qui a, pour la société wallisienne, la position d'ancien absolu. De fait, le premier-né, projeté au rang des anciens, a autorité sur toute sa fratrie. Il est appelé à succéder directement à son père dans l'exercice de l'autorité et ce quel que soit son sexe.

L'autre aspect de l'importance très nette que prennent la communion, puis le mariage, du premier-né d'un couple est le caractère massif de la participation des personnes ayant une quelconque relation avec les parents du communiant ou du marié. Cette contribution a un caractère nécessaire pour le premier-né, facultatif pour les autres enfants. Les explications de cet état de fait convergent chez mes informateurs. Les premières cérémonies de communion et de mariage qu'organise un couple sont l'occasion, pour l'ensemble des membres des côtés cérémoniels respectifs de l'homme et de la femme, de lui rendre de façon ostentatoire les prestations qu'il leur a, lui-même, fournies au cours des années de vie commune. Il s'agit donc d'un retour, massif et unitaire, dont les cérémonies du premier-né forment le cadre, mais dont elles ne sont pas nécessairement - et c'est là un fait intéressant - la condition.

Il peut arriver, en effet, que le mariage d'un puîné précède ou remplace celui de l'aîné. Il est alors significatif que les cérémonies préparées pour le puîné aient, dans ce cas, l'importance que celles de l'aîné auraient dû avoir. Ce qui apparaît alors comme un transfert répond en fait à un principe qui, généralement superposé à la primogéniture, lui est en fait disjoint. Si la position du premier-né suscite une attention particulière de ses parentèles lors des cérémonies le concernant, c'est aussi la participation importante et régulière du couple de ses parents aux prestations concernant les membres de leurs " côtés cérémoniels » kutuga qui est manifestée et restituée de façon différée, mais avec éclat. Ces jours de grand retour coïncident généralement avec les cérémonies de l'aîné, mais pas nécessairement. On voit bien alors que les rites de passage, de l'aîné comme des puînés, s'insèrent dans un circuit de prestations qui suit deux logiques superposées. D'une part est affirmée la distinction de l'ancienneté qui, à la génération des enfants, assure, en la personne du premier-né - en relation directe avec le côté paternel-, le renouvellement de la vie ainsi que la transmission de l'autorité et des responsabilités au sein de la parentèle plus ou moins étendue. D'autre part, c'est le couple des parents qui est distingué : formant une unité sociale efficace, il participe aux prestations concernant les côtés respectifs de chacun de ses membres, s'assurant ainsi, outre une place dans ces deux ensembles (côté homme et côté femme), la reconnaissance de cette place et celle qu'y trouve sa descendance ${ }^{20}$.

Ces deux niveaux d'organisation et de relations cérémonielles manifestent deux temporalités différentes et complémentaires. La différence réside dans le fait qu'elles sont prises en charge de façon contrastée par les maternels et les paternels. Premièrement, l'aîné, premier enfant d'un couple, ouvre, suite à la défloration de sa mère, puis la réclusion de ses couches, une nouvelle génération en relation à une terre et à un groupe social localisé et maternel. À ces responsabilités

20. La non-participation aux obligations cérémonielles villageoises par un couple, par exemple, le place de fait hors de sa communauté. S'il se dérobe aux obligations dites « familiales » (fakafamili), on ne peut parler d'exclusion, mais les conséquences sont plus graves encore en ce qu'il ne sera guère consulté en conseil de famille et que les droits de sa descendance sur le patrimoine du groupe (terres et titres) trouveront bien peu de défenseurs en cas de litige. 
spécifiques des maternels répondent, mais de façon secondaire, la reconnaissance et le don du nom par les paternels. Le premier enfant prend ainsi sa place - et repousse ses parents d'un cran - dans la succession des générations qui, à l'échelle de la communauté villageoise, prend forme dans la gradation des classes d'âge. Il s'agit, ici, de perpétuer l'organisation du village sous forme de maisonnées pérennes dont les couples mariés prennent en charge la vie matérielle et cérémonielle. Ainsi les villageois participent du renouvellement de la vie et des formes de circulation - conformation et dispersion des côtés cérémoniels et de leurs prestations - dont chaque maisonnée - et le couple responsable est le point de jonction. Génération après génération, de nouveaux vivants et de nouveaux morts occupent ces terres, deux côtés se rejoignant autour de chaque enfant pour ne plus former qu'un seul côté, le jour de son mariage, face à celui de son conjoint. Ainsi, autour de chaque premier-né, un couple de parents réalise dans sa descendance l'union de deux côtés cérémoniels qui, face à l'extérieur, ne feront désormais qu'un seul et même côté pour toute la fratrie. Ici, l'aîné, qu'assistera le temps venu son futur conjoint, prépare la succession de ses parents que la société valorise pour la capacité génésique dont ils ont fait preuve en procréant et la régénération de l'unité « maisonnée » qu'ils assurent - y compris par l'« adoption » et l'accueil de dépendants - , en y organisant une vie prospère et durable. En effet, c'est de la relation privilégiée du couple à Dieu et aux ancêtres (manu'ia), que la fertilité du sol et le bien être général des occupants de la maisonnée dépendent.

Deuxièmement, à cette dimension particulière de l'aînesse manifestée dans les cérémonies du cycle de vie, en relation à la fertilité d'un couple et d'une maisonnée, s'adjoint une autre dimension qui ne s'y superpose pas tout à fait. En l'aîné, plus qu'en tout autre enfant, se forme la jonction de deux parentèles, ossatures et noyaux de vastes côtés cérémoniels qui se mobilisent de façon symétrique, bien que différenciée, lors des rites de passage qui le concernent. Cette conjonction de relations maternelles et paternelles étendues en fait un être complet, ouvrant le chemin pour ses puînés. Cependant, ici, l'accentuation est nette du côté paternel. Une fois qu'il est reconnu par son père et ses paternels, la position éminente de l'aîné dans le vaste réseau de ces relations le désigne, quel que soit son sexe, comme successeur de son père, non tant comme responsable du groupe localisé que du groupe de descendance en relation aux ancêtres prestigieux sur lesquels s'adosse le statut. Ici, la relation de référence n'est plus celle du couple de parents, mais celle de la paire que forment le père et sa sœur - en premier lieu sa sœur aînée - en relation aux ancêtres paternels. Alors, l'aîné prend place parmi les «anciens» (mātu'a) qui, détenant l'autorité sur leur parentèle, président aux décisions la concernant. Comme ancien, il s'insère dans la gradation des statuts et du rang ${ }^{21}$ - statut social, et prestige personnel des ascendants et des collatéraux, position relative de ceux-ci au sein de leur groupe de descendance... - et dans l'ordonnancement de la société qui se mobilise lorsqu'il boit la coupe de kava royale pour sa première communion. Ce jour-là s'opère la reconnaissance formelle de son statut et de celui de sa parentèle, en présence du clergé et de la chefferie qui l'accueillent de plein droit dans la communauté chrétienne, lui rappelant ses devoirs envers ses anciens, ses puînés et Dieu qui veille au bon ordre du monde.

\section{Conclusion}

D'une façon assez classique, les rites de passage wallisiens assurent à l'enfant, puis à l'adulte qu'il deviendra, une existence sociale complète par un traitement rituel approprié de sa personne physique et sociale. Ils impliquent également l'agrégation, autour de lui, des « côtés cérémoniels » (kutuga) maternel et paternel. Dans tous les cas, la relation nécessaire entre un homme et une femme, mais aussi et surtout entre deux côtés cérémoniels, est soulignée. C'est elle qui permet la conception de l'enfant et qui se concrétise de façon formelle autour de lui.

Dans cette perspective, le mariage est la forme d'union la plus valorisée par la société wallisienne actuelle. Il crée, à partir d'un homme et d'une femme, une entité nouvelle et efficace pour la reproduction de la vie et le développement des relations sociales. Le lien entre les parents et leurs côtés respectifs prend tout son sens et toute sa dimension à la naissance du premier-né qui, à la fois, concrétise et justifie cette union. Les enfants " puînés » (muli, litt. " venir après, se mettre à la suite de quelqu'un ») suivent alors

21. La valorisation de la primogéniture se répercute dans la succession des générations de telle sorte que l'aînesse distingue également entre elles les branches généalogiques collatérales selon le rang de leurs fondateurs respectifs et sans considération pour l'âge de leurs représentants du moment. Le caractère structurant de l'aînesse dans la hiérarchie des groupes sociaux ainsi que dans l'organisation de la société wallisienne sort du champ volontairement restreint de cette analyse. Il est donc ici laissé de côté (pour son analyse détaillée, voir Chave-Dartoen, 2000). 
leur «aîné » (mu'a, litt. " précéder, aller en tête »). Chacun des puînés, important en luimême, n'aura cependant jamais le statut particulier de l'aîné, autour de qui les relations concernant les parents se sont nouées puisqu'il ouvre la descendance et manifeste l'impulsion initiale dans la succession entre deux générations.

Toutefois, ce qui est important ici, ce n'est pas tant les individus, bien que les considérations de statut personnel-autorité et prestige - ne soient pas insignifiantes, loin de là. Le caractère essentiel des rites de passage présentés est une dynamique qui agit à deux niveaux. D'une part, elle assure le flux réglé des prestations à travers la confrontation (fehaga'i) de côtés cérémoniels complémentaires qui se fondent à chaque génération pour se reconfigurer à la suivante. D'autre part, par cette confrontation de côtés, à la fois symétriques et différenciés, plus ou moins importants selon la position de la personne dans l'ensemble des relations qui la définissent avec ses ascendants, elle opère une distinction de rang et de statut sous la direction des anciens des deux côtés cérémoniels, mais aussi de ceux que la société se reconnaît, le "roi » hau et la « chefferie » kau'aliki, Dieu et le clergé.

Cette dynamique s'instaure ainsi en conformité avec les valeurs structurantes de la société wallisienne, mais aussi dans la nécessité constante de constituer et de travailler ces mêmes valeurs. À chaque occasion, pour chaque groupe, les relations sont évaluées, les anciens désignés, tandis que sont considérées leurs capacités à mobiliser leurs dépendants pour le prestige et le statut de tous. C'est à cette aune que sera attribuée la place des deux membres du couple et, au-delà, de leur descendance commune, dans les cérémonies à venir.

La procréation est donc ici fondamentale, mais la descendance directe n'est intéressante que dans une optique comparativement très réduite : transmissions de la vie et du statut s'inscrivent dans une circulation qui dépasse et intègre le couple en ce que, d'une part, elles relèvent du mouvement des humains et des prestations sur et dans les terres et, d'autre part, elles répondent à l'agencement spécifique des côtés cérémoniels dont chacun se trouve être à la jonction. Lors des rites de passage, deux temporalités comme deux types d'espace et de relations sont mis en conjonction. À un niveau local, le renouvellement de la société se fait par le relais qu'assurent les couples se succédant dans une maisonnée, la transmission de la vie étant assurée par les femmes en tant qu'épouses et mères en relation à leur terre natale et à leur communauté d'origine. Au niveau de l'unité que forme la société wallisienne, le renouvellement de la société s'appuie sur le relais que constituent, à chaque génération, les paires de germains de sexe opposé aînés, les femmes, en position de sœur cette fois, conférant le statut et garantissant la vie-même des neveux agnatiques en relation aux ancêtres prestigieux et à Dieu, c'est-à-dire en relation à la société dans sa forme largement déployée dans le temps et dans l'espace.

C'est à la jonction de ces deux niveaux d'organisation des relations et de ces deux formes de temporalités que les rites de passage étudiés ici procèdent à une jonction dont la personne focale est le support, mais dont ses parents, ses côtés cérémoniels et, au-delà, l'ensemble de la société sont, à des degrés divers opérateurs, responsables et bénéficiaires.

\section{BIBLIOGRAPHIE}

Bataillon Pierre (Monseigneur), 1932. Langue d'Uvea, grammaire-dictionnaire Uvea-français, suivi $d u$ dictionnaire français-uvea-anglais, Paris, Librairie orientale Paul Geuthner.

Bourdieu Pierre, 2001. Les rites d'institution, in Langage et pouvoir symbolique, Paris, Seuil, coll. PointsEssais.

Burrows Edwin G., 1937. Ethnology of Uvea (Wallis Island), Honolulu, Bernice P. Bishop Museum Bulletin 145.

Chave Sophie, 1992. La cérémonie du kava à Wallis (Polynésie occidentale), mémoire de DEA en Anthropologie sociale, Paris, EHESS.

—, 2000. Uvea (Wallis), une société de Polynésie occidentale, étude et comparaison, mémoire de doctorat en Ethnologie et Anthropologie sociale Paris, EHESS.

Henquel Joseph, s.d. Talanoa ki Uvea nei, manuscrit dactylographié, mission de Lano, Wallis.

TURNER Victor, 1969. Le phénomène rituel. Structure et contre-structure, Paris, PUF, coll. Ethnologies.

VAN GENNEP Arnold, 1909. Les rites de passage, Paris, Éditions A\&J (édition originale : E. Nourry).

VIALA Maxime, 1909. Les îles Wallis et Horn, Bulletin de la Société neuchâteloise de géographie 28, pp. 209-283. 